\title{
Newly Developed Anti-Dandruff Regimen, VB-3222, Delivers Enhanced Sensorial and Effective Therapeutic Benefits Against Moderate Adherent Dandruff
}

This article was published in the following Dove Press journal:

Clinical, Cosmetic and Investigational Dermatology

\author{
Suresh Sadhasivam (D) \\ Kalpna Garkhal' \\ Himanshi Singh' \\ Vishal Yadav (D) \\ Suresh Chawrai' \\ Mukesh Ramnane ${ }^{2}$ \\ Shilpi Jain (D) \\ Kabir Sardana $\left(1 D^{3}\right.$ \\ Shamik Ghosh' \\ 'Vyome Therapeutics Limited, New \\ Delhi I I0092, India; ${ }^{2}$ MS Clinical \\ Research Pvt. Ltd, Ulsoor, Bangalore \\ 560008, India; ${ }^{3}$ Department of \\ Dermatology, Dr RML Hospital and \\ PGIMER, New Delhi II000I, India
}

Background: Uninhibited proliferation of Malassezia spp., enhanced sebaceous gland activity and individual sensitivity are three prime etiological factors behind dandruff. For many dandruff sufferers, existing anti-dandruff products start yielding unsatisfactory results after a few cycles of use. This observation made us explore the physical and biological environment of the host and exploit the specific type of lipid dependence of Malassezia spp. for their survival. A shampoo formulation (product code VB-3222) was developed to address the shortcomings of existing therapy.

Purpose: Evaluating efficacy of VB-3222 in comparison to marketed products through in vitro assays and subsequently demonstrating its advantages in a clinical study.

Methods: VB-3222 was developed with a derivative of medium chain fatty acid (MCFA) and zinc pyrithione and compared against marketed comparators by in vitro time kill assay. Subsequently, VB-3222 shampoo was tested in a 21-day clinical trial on 25 moderate dandruff subjects to evaluate local safety and efficacy.

Results: VB-3222 in all in vitro cases demonstrated significantly better fungicidal activity than its marketed comparators. In the clinical trial, VB-3222 was well tolerated in all subjects and imparted consistent reduction of the ASFS (adherent scalp flaking score) and the pruritus score. At days 7 and $21,55 \%$ and $90 \%$ reduction in the ASFS in comparison to treatment initiation and $50 \%$ and $95.5 \%$ reduction in the pruritus score were observed.

Conclusion: The increased efficacy of VB-3222 over comparator products in vitro, and the dramatic reduction $(>90 \%)$ in ASFS and pruritis in subjects within 21 days of use with excellent tolerability and sensorial profile, positions VB-3222 as the new generation treatment for adherent dandruff.

Clinical Trial Registration No: CTRI/2018/05/013567.

Keywords: anti-dandruff shampoo, Malassezia spp., potentiator, dandruff, seborrheic dermatitis

\section{Introduction}

Seborrheic dermatitis (SD) and dandruff are common skin problems that are widely prevalent. SD is predominantly seen in three different age groups, children within 3 months of birth, teenagers and adults aged 40-60 years. SD affects the scalp, face, chest and the other sebum-rich areas in the skin. SD is characterized by flaking, scaling, pruritus and pronounced inflammation with erythema. Dandruff is usually
Correspondence: Shamik Ghosh

$\checkmark$ yome Therapeutics Limited, 465

New Delhi II0092, India

Email shamik.ghosh@vyometx.com

Kabir Sardana

Department of Dermatology, Dr. RML

Hospital and PGIMER, New Delhi

I I000I, India

Email article.sardana@gmail.com 
limited to the scalp, cause scaling and itching with mild or no inflammation. ${ }^{1-5}$ Increased proliferation of Malassezia spp., enhanced sebaceous gland activity and individual sensitivity are considered prime reasons for dandruff or SD. ${ }^{6}$ Malassezia spp. are known to be the causal agents for dandruff and SD for decades but the severity depends on the inherent immunological response of the individuals and the nature of the stratum corneum integrity. ${ }^{7}$ Most of the species in genus Malassezia are lipophilic and are present in the skin of warm-blooded animals and humans. The Malassezia genus comprises 14 different species and recently one new species Malassezia arunalokei is additionally included under this genus. ${ }^{8}$ The genomic sequence analysis indicates that the Malassezia genus does not possess the crucial gene required for fatty acid synthesis; instead, it has multiple variants of lipase and phospholipase genes. ${ }^{9-11}$ Malassezia secretes lipases and hydrolases which act on human sebum and releases diglycerides, unsaturated and saturated fatty acids on the scalp; most of the saturated long chain fatty acids (LCFA) such as palmitic acid are consumed by the fungus itself for its own growth on the scalp, and in sensitive skin with major alterations in barrier integrity some of these unsaturated fatty acids penetrate the stratum corneum and induce inflammation and enhance abnormal keratinization. ${ }^{10}$ There are many anti-dandruff shampoo and leave-on formulations available on the market to treat dandruff. Most of these formulations have one or more of the following active ingredients such as ketoconazole, zinc pyrithione and climbazole. ${ }^{12-14}$ They all have shown proven efficacy in vitro and in controlled clinical trial conditions. But these formulations face multiple challenges in alleviating dandruff suffering, and a significant number of people remain dissatisfied with the quality or the extent of relief these formulations provide.

There are two major factors observed in anti-dandruff product performance. Firstly, in most cases, though these anti-fungal agents take care of the initial symptoms by removing Malassezia from the scalp, the inherent lipid imbalance in the microbial niche has never been taken care of. The stronger the agents are, the harsher are their footprints on the scalp stratum corneum. These deteriorated scalp environments predispose to an aggravated inflammatory response which may lead to a severe form of dandruff or SD.

Secondly, the sensorial properties of these anti-fungal formulations, mainly shampoos, are so unattractive that in real life, medium to long-term adherence to the advised therapeutic regime becomes a challenge for the patients.
This further leads to a vicious cycle of less compliance, more relapse and lesser therapeutics options in the arsenal of the physicians in progressively worsening cases. This led us to design the present sets of study to determine the effect of a select medium chain fatty acid (MCFA) derivative which could eventually take care of some limitations of the existing formulations for the treatment of moderate dandruff.

A careful observation of product ingredient labels suggests that many of them inadvertently use LCFA or their derivatives in the formularies. LCFA and related derivatives enable the fungus to get nutrition and maintain cellular integrity while reducing the efficacy of the marketed formulation. This context indulges us to conceive an idea where LCFA and their derivatives in the formularies are being replaced by a select derivative of a MCFA to deprive the fungus of its coveted nutrition. Because of its ability to change the composition of the fungal niche at the molecular level this platform technology is named MRT (Molecular Replacement Therapeutics). MRT not only exploit the obligate nutrient need of the fungus but also potentiate existing agents to work more effectively by destabilizing the fungal cell structure.

In view of these points we undertook an in vitro time kill study followed by a clinical study to evaluate the efficacy of Vyome in-house shampoo formulation, product code no. VB-3222, for the treatment of moderate adherent dandruff. The primary objective of the study was to evaluate the efficacy of the test product for the adherent scalp flaking score (ASFS) and pruritus score (Investigators' Global Assessment, IGA) at day 21 in comparison to baseline. Secondary objectives were as follows: 1) to evaluate the efficacy of the test product in reduction of the ASFS and pruritus, with reference to the number of washes, at all time points in comparison to baseline; 2) to evaluate the efficacy of the test product in reduction of flaking (non-adherent dandruff), with reference to the number of washes, at all time points in comparison to baseline; 3) to evaluate the safety of the test product; and 4) to evaluate the product acceptance analysing sensorial parameters.

\section{Materials and Methods}

\section{Investigational Product}

VB-3222 anti-dandruff shampoo formulation was developed at Vyome Therapeutics Limited by including a synthetic derivative of a MCFA (MRT, considered a GRAS excipient) to a mixture of zinc pyrithione (ZPT) dissolved in a wellaccepted topical formulation base to obtain a smooth, rich 
and free from undissolved solid material shampoo. VB-3222 was packaged into uniform transparent plastic bottles with a small opening. The detailed composition of the shampoo is as follows: sodium lauryl ether sulphate, sodium lauryl sulphate, water, cocamidopropyl betaine, acrylate copolymer, MRT-3\%, laureth-4, amodimethicone emulsion, cocomide MEA, zinc carbonate, zinc pyrithione- $1 \%$, fragrance, panthenol, bromelain enzyme, cetyl alcohol, laureth-23, magnesium carbonate, sodium citrate, hydrochloric acid, sodium hydroxide, guar hydroxypropyl trimonium chloride, menthol, chloro-methylisothiazolinone and methylisothiazolinone.

\section{In Vitro Biology Assay Materials}

Sabouraud dextrose broth (HiMedia, Mumbai) with 2\% olive oil, Sabouraud dextrose agar (HiMedia, Mumbai) with 2\% olive oil, Malassezia furfur MTCC 1374 (procured from Microbial Type Culture Collection and Gene Bank MTCC, Chandigarh) and marketed anti-dandruff shampoos (Table 1) were procured from local medical shops.

\section{Time Kill Assay Method}

In the time kill experiment, fungicidal activity of the test and reference formulations was determined by adding the 10 -fold diluted antifungal formulations in Sabouraud dextrose broth with $2 \%$ olive oil containing a stipulated number of viable $M$. furfur cells. Subsequently, at various time points of $0,1,6$ and $24 \mathrm{~h}, 50 \mu \mathrm{L}$ of the sample was drawn from each representative tube (test and reference), diluted and spread over the surface of a Petri plate containing Sabouraud dextrose agar (HiMedia) with $2 \%$ olive oil. ${ }^{15,16}$ Anti-dandruff shampoos were 10 times diluted in sterile Milli-Q water, then $100 \mu \mathrm{L}$ of this $10 \%$ shampoo suspension was mixed with 900 $\mu \mathrm{L}$ of $M$. furfur culture suspension $\left(10^{6}-10^{7} \mathrm{CFU} / \mathrm{mL}\right)$ prepared in Sabouraud dextrose broth with $2 \%$ olive oil in a 2-mL microfuge tube (in the final mixer the formulation was subjected to 100 times of dilution). The total mixture

Table I Active Ingredients of Anti-Dandruff Shampoo Formulations Used in the Time Kill Experiment

\begin{tabular}{|c|c|c|}
\hline S. No & $\begin{array}{l}\text { Anti-Dandruff } \\
\text { Shampoos }\end{array}$ & Active Ingredients \\
\hline I & $\begin{array}{l}\text { VB-3222 (Product } \\
\text { Code) }\end{array}$ & Zinc pyrithione and MRT \\
\hline 2 & Shampoo A & Zinc pyrithione \\
\hline 3 & Shampoo B & $\begin{array}{l}\text { Climbazole and Melaleuca } \\
\text { leucadendron cajeput oil }\end{array}$ \\
\hline 4 & Shampoo C & Zinc pyrithione and ketoconazole \\
\hline
\end{tabular}

( $1 \mathrm{~mL}$ ) was incubated at $32^{\circ} \mathrm{C}$ in a tube rotator. After 1,6 and $24 \mathrm{~h}$, cells are plated after serial dilution. Cells were plated in SDA plates containing $2 \%$ olive oil in triplicate and allowed to grow for 3-4 days at $32{ }^{\circ} \mathrm{C}$.

\section{Clinical Trial Design}

This study was an open label, single arm, monocentric, safety and efficacy study. Protocol makes the requirement of good clinical practices, and Declaration of Helsinki. Ethical approval for this study was obtained from an independent ethics committee (Clinicom, Bangalore) before the initiation of the clinical trial. Healthy males or females aged 18-65 years with a clinical diagnosis of moderate adherent dandruff of the scalp were recruited for the study to monitor the effects. Subjects who satisfied study inclusion and exclusion criteria were enrolled after obtaining written informed consent. A total of 25 (24+1 additional subject as anticipatory compensation) adult male and female subjects had been enrolled in the study. A wash-in period of 2 weeks had been conducted from visit 1 (day -15 ) to visit 2 (day 1). The subjects had been instructed to use only the additional product, neutral shampoo, during the wash-in period. On visit 2 (day 1, baseline) the subjects had been provided with the test product (VB-3222) and had been instructed to use the same thrice weekly during the study period.

\section{Product Efficacy by Dermatologist Assessment}

The adherent scalp flaking score (ASFS) ${ }^{17,18}$ was evaluated by dividing each subject's scalp into eight zones and each section of the scalp was assessed for the presence of dandruff flakes that were adhering to the scalp skin using a 0-10 scale ( 0 means no flakes and 10 means heavy flaking). Loose flakes in the hair were not considered in the grading. The final or total ASFS was determined by adding the grades for all 8 zones on the scalp, which renders a possibility of a score between 0 and 80 . For representation purposes, the subject score was categorized as 1=Mild (16-24), 2=Moderate (25-34) and 3=Severe (35-80).

The IGA for pruritus score was evaluated on a scale where $0=$ no pruritus, $1=$ Present with mild discomfort, 2=Appreciable discomfort which interferes with daily activities and $3=$ Extreme discomfort.

\section{Product Safety by Dermatological Assessment}

The dermatological assessment form for application site reaction was completed by a dermatologist where scores $(0=$ None, 
1=Mild, 2=Moderate, 3=Severe, 4=Very severe) are used for parameters like dryness, scaliness and oiliness. The subject assessment form for application site reaction was completed by subjects, where $0=$ None, $1=$ Mild, $2=$ Moderate, $3=$ Severe and $4=$ Very severe was used for parameters like eye stinging and tingling.

\section{Statistical Methods}

Demographic characteristics and results of the study were summarized using mean \pm standard deviation (SD) for continuous variables and frequency and percentages for categorical variables. Significance was assessed at 5\% level of significance. i.e. $p \leq 0.05$. Statistical figures are at the $95 \%$ confidence level: comparative test evaluation was done using Student's $t$-test to compare efficacy in between time points.

\section{Results}

\section{In-vitro Biology}

The time kill experiment result indicates that compared to other shampoo formulations the in-house developed formulation, VB-3222, was very effective in exhibiting its fungicidal ability within a few hours of treatment (Figure 1). At the end of $6 \mathrm{~h}, \mathrm{VB}-3222$, anti-dandruff shampoo containing $1 \%$ ZPT (zinc pyrithione) with 3\% of MRT (potentiator), has shown more than $3 \log$ reductions, whereas other market formulations have shown less than $2 \log$ reductions. The difference in killing potential of VB-3222 vis-à-vis other marketed anti-dandruff shampoo formulations (including a formulation containing zinc pyrithione and ketoconazole) was statistically significant $(p<0.001)$.

\section{Dermatological Assessment for Efficacy} Adherent Dandruff Score (ASFS) Mean Percent Value

The population was at a moderate adherent dandruff level aligned with the inclusion criteria. A statistically significant and progressive improvement in the reduction in the level of adherent dandruff was observed at all time points of evaluation in comparison to the baseline. The improvement was statistically significant even from very first follow-up visit on day 5 after completion of just 2 hair washes. Moreover, about $86.36 \%$ of the study population showed improvement in reduction of ASFS on day 5. The mean adherent dandruff level reached a negligible score on day 9 after 4 washes. However, as anticipated, the complete riddance of dandruff was not seen in the study population. At the last visit, the population mean ASFS score remained 3.73 \pm 3.56 . 100\% of

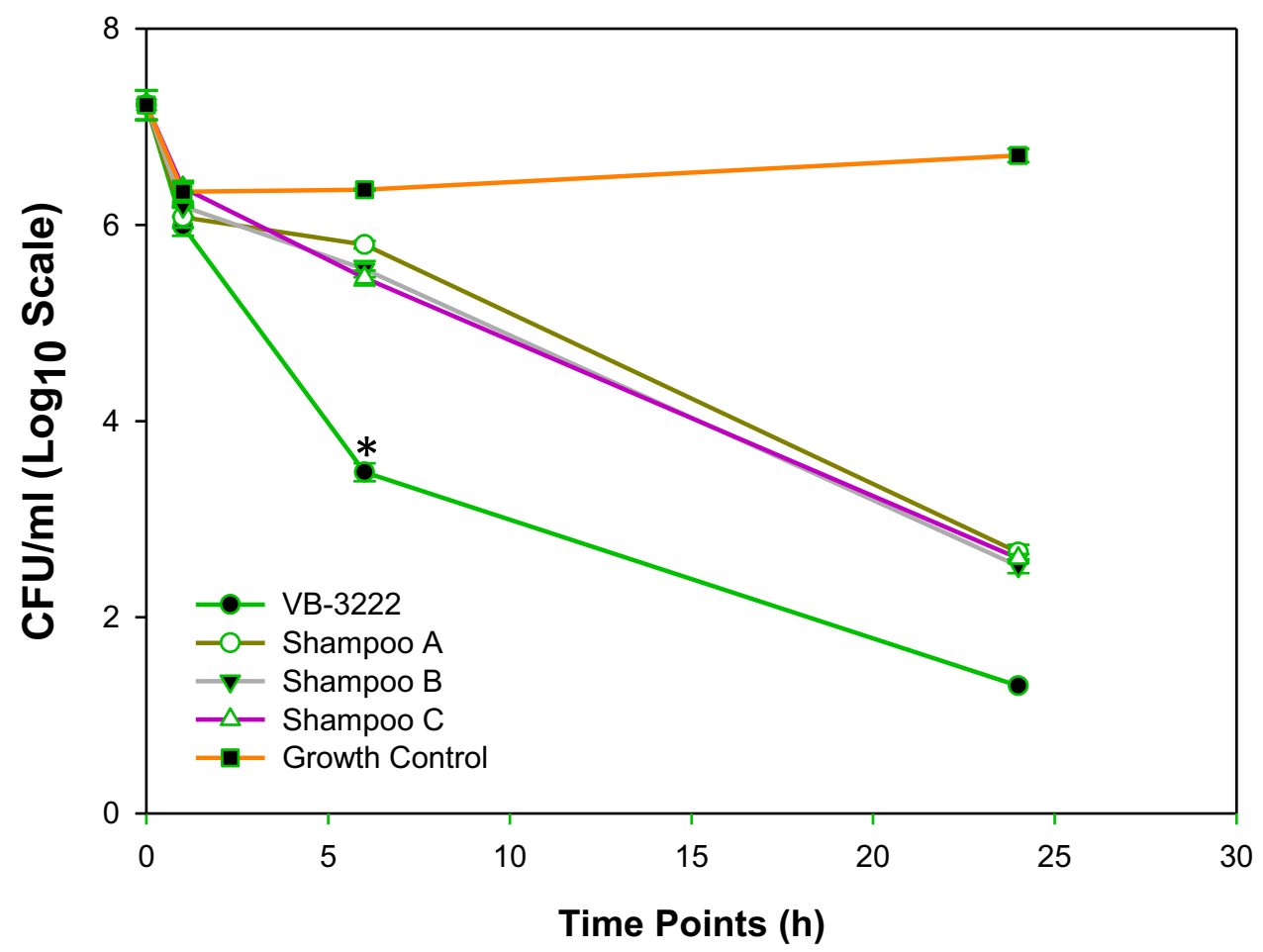

Figure I Antifungal efficacy of anti-dandruff shampoo formulations tested against Malassezia furfur MTCC I374 by time kill experiment. Time kill experiment of different anti-dandruff shampoo products (VB-3222 and Shampoo A, B and C) and growth control plotted in a graph. At time points 0 , I, 6 and 24 h, number of remaining viable $M$. furfur counts $\left(\mathrm{CFU} / \mathrm{mL}\right.$ in $\log _{10}$ scale). Each data point corresponds to the mean value of 3 replicates $\left({ }^{*} p=<0.00 \mathrm{I}\right)$. 
the population showed improvement on the ASFS score from day 7 onwards in comparison to the baseline (Figure 2). This improvement was sustained until the end of the study.

\section{Pruritus Mean Percent Value}

The pruritus score was obtained at baseline and days 5, 7, 9, 14, 16 and 21. The pruritus scores at various time points were represented in a percentage scale by considering the baseline mean value as $100 \%$. Statistically significant reductions were observed (Figure 3 ) in pruritus at all time points of the evaluation in comparison to baseline. About $50 \%$ of the population showed improvement in pruritus on day $5,77.28 \%$ of the population on day $7,95.45 \%$ of the population from day 9 to day 16 , and $100 \%$ of subjects showed improvement in pruritus at the end of the study in comparison to baseline.

\section{Flaking Score}

Along with adherent scaling, dandruff also results in nonadherent scaling which has been evaluated under flaking severity. On a 10-point scale, the population mean score was 6.41 at the beginning of the study. Even at the first follow-up visit, in response to 2 hair washes there was a significant reduction in the mean score (Figure 4). There was nearly 50\% improvement in QoL in 1 week and about $88 \%$ improvement in QoL at the end of the study.

\section{Sensorial Assessment}

Use of VB-3222 improved several scalp and hair related sensorial parameters. Scalp dryness was reduced consistently throughout early days of the study and no dryness was observed at day 16 in $100 \%$ of subjects and it was sustained until the end of the study, $81.25 \%$ of subjects showed improvement in hair brittleness, $81.82 \%$ of subjects showed improvement in hair shine, $90.91 \%$ of subjects showed improvement in hair softness and $78.95 \%$ of subjects showed improvement in hair detangling efficacy (Table 2). According to the self-assessment of the subjects, significant improvements were observed in the reduction of itching, flaking dandruff during the study period in comparison to baseline. At the end of the study, above $70 \%$ of the population showed reduction in itching and flaking dandruff in comparison to baseline.

\section{Safety Assessments}

One adverse event was recorded during the study, the subject had a mild-intensity accident which was not related to the study. The same subject was given concomitant medication for wound pain. In dermatological assessment, 12 subjects (8 mild, 3

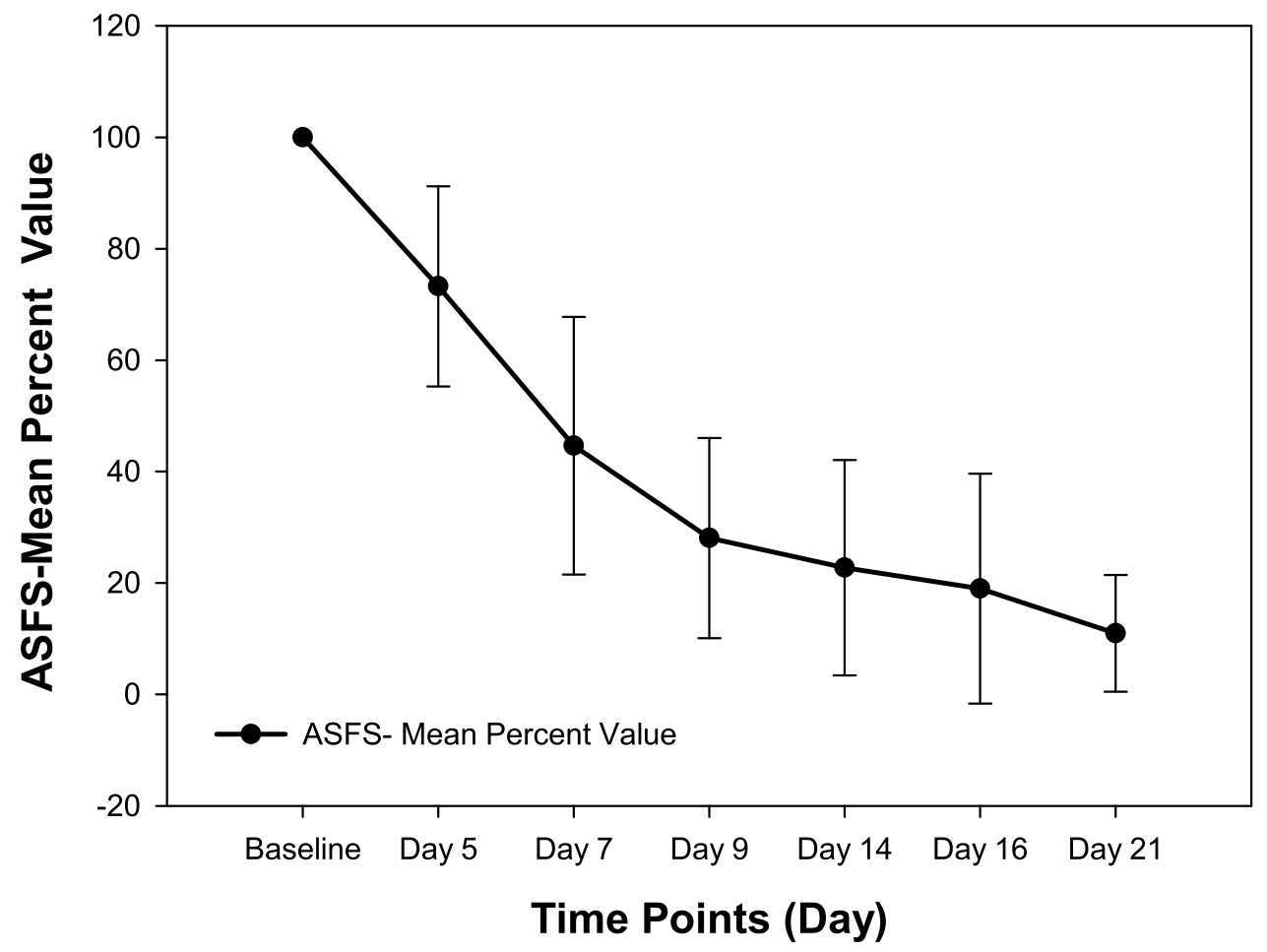

Figure 2 Reduction in mean percent values of the adherent dandruff flaking score (ASFS) post application of VB-3222. Reduction in the level of adherent dandruff is observed at all time points (baseline, days 5, 7, 9, 14, I6 and 21) of evaluation in comparison to the baseline. Each data point corresponds to the percent mean value of ASFS collected from 22 subjects $(n=22, \pm S D)(p=<0.0001)$. 


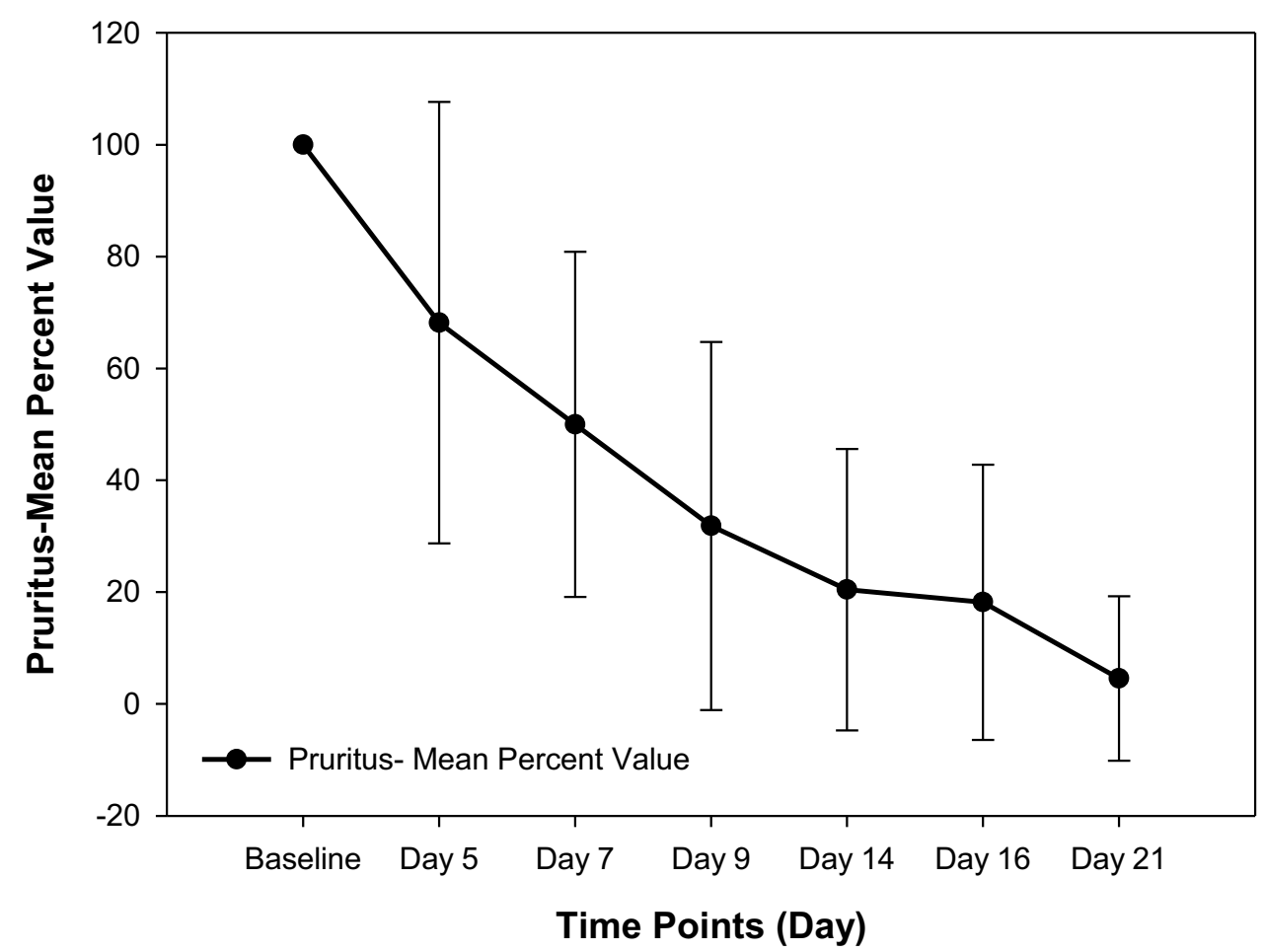

Figure 3 Reduction in mean percent values of the pruritus score post application of VB-3222. Reduction in the level of pruritus is observed at all time points (baseline, days $5,7,9,14,16$ and 21 ) of evaluation in comparison to the baseline. Each data point corresponds to the percent mean value of pruritus score from 22 subjects $(n=22, \pm S D)(p=<0.0001)$.

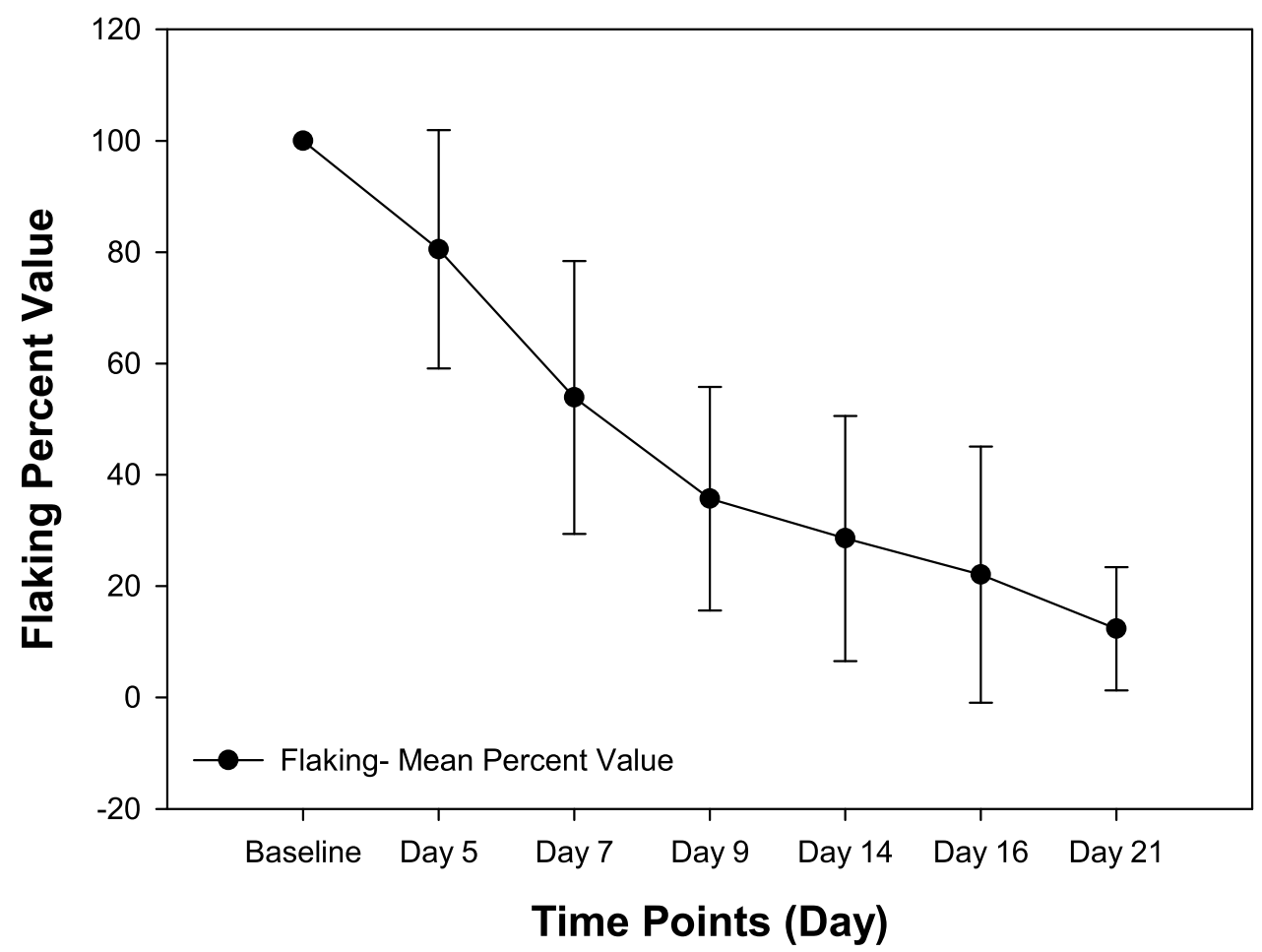

Figure 4 Reduction in mean percent values of the flaking score post application of VB-3222. Reduction in the level of flaking score is observed at all time points (baseline, days 5, 7, 9, 14, 16 and 21) of evaluation in comparison to the baseline. Each data point corresponds to the percent mean value of flaking score from 22 subjects $(n=22, \pm S D)(p=<0.0001)$. 
Table 2 Sensorial Assessment

\begin{tabular}{|c|c|c|c|c|c|c|c|c|}
\hline Scalp Condition & Visit & Baseline & Day 5 & Day 7 & Day 9 & Day 14 & Day 16 & Day 21 \\
\hline \multirow[t]{2}{*}{ Scalp Condition Dryness } & Mean $\pm S D$ & $2.05 \pm 1.13$ & $1.77 \pm 1.02$ & $1.32 \pm 0.78$ & $1.18 \pm 0.5$ & $1.09 \pm 0.29$ & $I \pm 0$ & $\mathrm{I} \pm 0$ \\
\hline & $p$ value & - & $0.055^{*}$ & $0.0004^{* * *}$ & $0.0003 * * *$ & $0.0005 * * *$ & $0.0002^{* * *}$ & $0.0002^{* * *}$ \\
\hline \multirow[t]{2}{*}{ Brittleness of hair } & Mean $\pm S D$ & $1.95 \pm 0.72$ & $1.82 \pm 0.66$ & $1.59 \pm 0.59$ & $1.55 \pm 0.5 \mid$ & $1.32 \pm 0.48$ & $1.32 \pm 0.48$ & $1.27 \pm 0.46$ \\
\hline & $p$ value & - & $0.0829 *$ & $0.0079 * *$ & $0.0038^{* *}$ & $0.0002 * * *$ & $0.0002^{* * *}$ & $<0.0001 * * *$ \\
\hline \multirow[t]{2}{*}{ Hair shine } & Mean $\pm S D$ & $2.36 \pm 0.49$ & $2.4 I \pm 0.5$ & $3.05 \pm 0.65$ & $3.23 \pm 0.61$ & $3.36 \pm 0.66$ & $3.55 \pm 0.6$ & $3.55 \pm 0.74$ \\
\hline & $p$ value & - & 0.3287 & $<0.0001 * * *$ & $<0.0001 * * *$ & $<0.000 I^{* * *}$ & $<0.000 I^{* * *}$ & $<0.0001 * * *$ \\
\hline \multirow[t]{2}{*}{ Hair softness } & Mean $\pm S D$ & $2.4 I \pm 0.73$ & $2.64 \pm 0.66$ & $3.05 \pm 0.79$ & $3.36 \pm 0.58$ & $3.45 \pm 0.6$ & $3.5 \pm 0.6$ & $3.64 \pm 0.58$ \\
\hline & $p$ value & - & $0.0566^{*}$ & $0.0005^{* * *}$ & $<0.0001 * * *$ & $<0.000 I^{* * *}$ & $<0.000 I^{* * *}$ & $<0.0001 * * *$ \\
\hline \multirow[t]{2}{*}{ Hair detangling } & Mean $\pm S D$ & $2.77 \pm 0.92$ & $2.64 \pm 0.95$ & $2.5 \pm 0.86$ & $2.36 \pm 0.79$ & $2.32 \pm 0.89$ & $2.23 \pm 0.8 \mathrm{I}$ & $1.91 \pm 0.68$ \\
\hline & $p$ value & - & 0.1858 & $0.0303 * *$ & $0.0038 * *$ & $0.0018^{* *}$ & $0.0003^{* *}$ & $<0.000 I^{* *}$ \\
\hline
\end{tabular}

Note: *Suggestively significant at $\mathrm{p}$-value $\leq 0.10 ; * *$ Statistically significant at $\mathrm{p}$-value $\leq 0.05 ; * * *$ Statistically highly significant at $\mathrm{p}$-value $\leq 0.00 \mathrm{I}$.

moderate and 1 severe) were observed with dryness at day 1 , which subsided to 9 subjects at day 7 . The dryness was completely resolved at the end of the study without any intervention. All 25 subjects ( 3 mild, 15 moderate and 7 severe) were observed to have scaling at day 1 . The reaction was noted in 13 subjects with mild severity, at day 21 . One subject was observed with mild oiliness at day 1 , and at day 5 , which was resolved at day 7. None of the subjects had erythema, allergic reaction and folliculitis throughout the study.

In subject assessment, one subject perceived mild eye stinging during lathering at day 1 and at day 5 . Eye stinging was the event of any unintentional eye contact. One subject perceived severe eye stinging at day 1 while another subject reported mild eye stinging at day 5 . No further reactions were recorded during the study period. 3 subjects reported mild tingling at day 1 and 1 subject reported mild tingling at day 5. None of the subjects had burning sensation, allergic reactions and eruptions/boils throughout the study. Overall, the product was considered safe.

\section{Product Tolerance}

The product was overall well tolerated as there were no untoward events.

\section{Discussion}

Our in vitro data suggest that marketed ZPT alone or ZPT plus ketoconazole may take significantly longer time to kill a fungal cell than VB-3222, the MRT potentiated ZPT formulation. Though the exact mechanism of potentiation is still being investigated, it is quite obvious VB-3222 acted much faster than conventional shampoos in vitro. Even in a human trial, VB-3222 delivered highly significant benefits in early days of treatment just after 2 applications. Within 4 applications adherent flaking in the scalp went down to almost negligible levels and these benefits sustained until the end of the study. The pruritus was improved consistently over the treatment period, within a week it had reduced significantly in $>75 \%$ of the subjects. To achieve this early efficacy the sensorial properties of VB-3222 were never sacrificed. Rather, by careful selection of conditioning agents it was demonstrated even in an anti-dandruff product with measurable enhancement of subject experience that it is possible to improve scalp dryness, hair brittleness, hair shine and hair detangling efficacy.

As stated earlier, we were also very selective in selection of the anti-fungal active zinc pyrithione (ZPT) for the study. One of our objectives was to enhance the efficacy and potency of the existing agents for dandruff so that the physicians did not need to use the FDA monographed drug products at the outset for the treatment of moderate dandruff. ZPT is the most common anti-dandruff agent in use. It is fungistatic in nature. It acts as a copper ionophore and it inhibits fungal growth through inactivation of iron sulphur protein within the fungal cell. ${ }^{19}$ On the other hand, ketoconazole mainly acts on enzymes of the ergosterol synthesis pathway. ${ }^{20}$ But their activity depends upon the local concentration of active drug in the vicinity of the fungus and the metabolic state of the fungus. Being a wash-off formulation, the deposition of actives in the appropriate amount at relevant layers may take a few rounds of washes for a visible reduction in fungal load on the scalp. However, our clinical study 
has certain limitations. It did not include a comparator arm. This study was exploratory in nature and lacked a placebo arm/comparative arm in the clinical trial. This pilot study can be a way forward to an elaborate future study with a comparative arm of marketed products in a larger population. However, all of the parameters were rigorously compared with the baseline observation. The goal was to see whether this powerful combination of anti-dandruff agent, MRT and select conditioning agent provided the subjects with early relief from flaking and itching with desired sensory benefits.

\section{Limitation of Study}

The primary purpose of this study was to evaluate the safety and efficacy of newly developed anti-dandruff shampoo VB3222 using medium chain fatty acids in the formulation. Some limitations of the study should be noted while analysing our results. This study was exploratory in nature and lacked a placebo arm/comparative arm in the clinical trial. It was planned as a pilot to an elaborate future study with a comparative arm of marketed products in a larger population.

\section{Conclusion}

In our study, we found that a potentiated ZPT shampoo, VB-3222, provides an effective, safe and sensorial attractive formulation for treatment of moderate adherent dandruff ensuring strong compliance.

\section{Data Sharing Statement}

The authors intend to share individual deidentified participant data for the text, tables and figures in the manuscript and study protocol (soft copy, PDF and password protected); however, a proposal should be submitted within 3 months of publication and data requestor will need to sign a data access agreement. Data will be available until 1 month after access.

\section{Disclosure}

SS, KG, HS, VY, SJ and SG are employees of Vyome Therapeutics Limited and SG holds stock in Vyome Therapeutics Limited. SC is an ex-employee and holds equity in Vyome Therapeutics Limited. Vyome Therapeutics Limited having patent application (patent no.: 10232047; date of patent: March 2019). KS is a subject expert on the topic with clinical expertise on seborrhoeic dermatitis. MR is a clinical investigator of the study and consultant to MS Clinical Research Private Limited. Vyome Therapeutics Limited provided the financial support for the study. The authors report no other conflicts of interest in this work.

\section{References}

1. Borda LJ, Wikramanayake TC. Seborrheic dermatitis and dandruff: a comprehensive review. J Clin Investig Dermatol. 2015;3(2):1-22.

2. Gupta AK, Madzia SE, Batra R. Etiology and management of seborrheic dermatitis. Dermatology. 2004;208(2):89-93. doi:10.1159/000076478

3. Del Rosso JQ. Adult seborrheic dermatitis: a status report on practical topical management. J Clin Aesthet Dermatol. 2011;4(5):32-38.

4. Sampaio AL, Mameri AC, Vargas TJ, et al. Seborrheic dermatitis. An Bras Dermatol. 2011;86(6):1061-1071. doi:10.1590/S036505962011000600002

5. Schwartz RA, Janusz CA, Janniger CK. Seborrheic dermatitis: an overview. Am Fam Physician. 2006;74(1):125-130.

6. DeAngelis YM, Gemmer CM, Kaczvinsky JR, et al. Three etiologic facets of dandruff and seborrheic dermatitis: malassezia fungi, sebaceous lipids, and individual sensitivity. J Investig Dermatol Symp Proc. 2005;10(3):295-297. doi:10.1111/j.1087-0024.2005.10119.x

7. Harding CR, Moore AE, Rogers SJ, et al. Dandruff: a condition characterized by decreased levels of intercellular lipids in scalp stratum corneum and impaired barrier function. Arch Dermatol Res. 2002;294(5):221-230. doi:10.1007/s00403-002-0323-1

8. Honnavar P, Prasad GS, Ghosh A, et al. Malassezia arunalokei sp. nov., a novel yeast species isolated from seborrheic dermatitis patients and healthy individuals from India. J Clin Microbiol. 2016;54(7):1826-1834. doi:10.1128/JCM.00683-16

9. Xu J, Saunders CW, Hu P, et al. Dandruff-associated Malassezia genomes reveal convergent and divergent virulence traits shared with plant and human fungal pathogens. Proc Natl Acad Sci U S A. 2007;104(47):18730-18735. doi:10.1073/pnas.0706756104

10. Dawson TL Jr. Malassezia globosa and restricta: breakthrough understanding of the etiology and treatment of dandruff and seborrheic dermatitis through whole-genome analysis. J Investig Dermatol Symp Proc. 2007;12(2):15-19. doi:10.1038/sj.jidsymp.5650049

11. DeAngelis YM, Saunders CW, Johnstone KR, et al. Isolation and expression of a Malassezia globosa lipase gene, LIP. J Invest Dermatol. 2007;127(9):2138-2146. doi:10.1038/sj.jid.5700844

12. Trueb RM. Shampoos: ingredients, efficacy and adverse effects. $J$ Dtsch Dermatol Ges. 2007;5(5):356-365. doi:10.1111/ddg.2007.5.issue-5

13. Ranganathan S, Mukhopadhyay T. Dandruff: the most commercially exploited skin disease. Indian J Dermatol. 2010;55:130-134. doi:10.4103/0019-5154.62734

14. Narshana M, Ravikumar P. An overview of dandruff and novel formulations as a treatment strategy. Int J Pharm Sci Res. 2018;9(2):417-431.

15. MacGowan AP, Wootton M, Hedges AJ, et al. A new time-kill method of assessing the relative efficacy of antimicrobial agents alone and in combination developed using a representative beta-lactam, aminoglycoside and fluoroquinolone. J Antimicrob Chemother. 1996;38 (2):193-203. doi:10.1093/jac/38.2.193

16. Roques C, Brousse S, Panizzutti C. In vitro antifungal efficacy of ciclopirox olamine alone and associated with zinc pyrithione compared to ketoconazole against Malassezia globosa and Malassezia restricta reference strains. Mycopathologia. 2006;162(6):395-400. doi:10.1007/s11046-006-0075-0

17. Schwartz JR, DeAngelis YM, Dawson TL Jr. Dandruff and seborrheic dermatitis: a head scratcher. In: Evans T, Wickett R, editors. Practical Modern Hair Science. 1st ed. Carol Stream: Allured; 2012:389-413.

18. Bacon RA, Mizoguchi H, Schwartz JR. Assessing therapeutic effectiveness of scalp treatments for dandruff and seborrheic dermatitis, part 1: a reliable and relevant method based on the adherent scalp flaking score (ASFS). J Dermatolog Treat. 2014;25(3):232-236. doi:10.3109/09546634.2012.687089 
19. Reeder NL, Kaplan J, Xu J, et al. Zinc pyrithione inhibits yeast growth through copper influx and inactivation of Iron-Sulfur proteins. Antimicrob Agents Chemother. 2011;55(12):5753-5760. doi:10.1128/AAC.00724-11
20. Van Tyle JH. Ketoconazole mechanism of action, spectrum of activity, pharmacokinetics, drug interactions, adverse reactions and therapeutic use. Pharmacotherapy. 1984;4(6):343-373. doi:10.1002/ j.1875-9114.1984.tb03398.x

\section{Publish your work in this journal}

Clinical, Cosmetic and Investigational Dermatology is an international, peer-reviewed, open access, online journal that focuses on the latest clinical and experimental research in all aspects of skin disease and cosmetic interventions. This journal is indexed on CAS.

Submit your manuscript here: https://www.dovepress.com/clinical-cosmetic-and-investigational-dermatology-journal
The manuscript management system is completely online and includes a very quick and fair peer-review system, which is all easy to use. Visit http://www.dovepress.com/testimonials.php to read real quotes from published authors. 